\title{
Macular Hole Surgery in the Case of Wet Age-Related Macular Degeneration Treated with Intravitreal Aflibercept
}

\author{
Agnieszka Nowosielska
}

Ophthalmology and Eye Surgery Clinic, Private Ophthalmic Center, Warsaw, Poland

\section{Keywords}

Macular hole $\cdot$ Vitrectomy $\cdot$ Wet age-related macular degeneration $\cdot$ Choroidal neovascularization · Anti-VEGF treatment · Eylea · Aflibercept

\begin{abstract}
A 72-year-old male treated with aflibercept (Eylea) due to choroidal neovascularization (CNV) in his left eye developed a full-thickness macular hole $(\mathrm{MH})$ after the second injection. The occurrence of $\mathrm{MH}$ in his left eye resulted in further visual acuity deterioration. As a consequence of having developed the $\mathrm{MH}$, the patient was operated on. Phacoemulsification and vitrectomy with internal limiting membrane peeling and 20\% SF6 tamponade were performed. $\mathrm{MH}$ closure was achieved and best corrected visual acuity improved to the extent allowed by CNV. The patient continued anti-VEGF treatment with Eylea due to wet age-related macular degeneration in his left eye. The observation period of 2 years has been uneventful and visual acuity of 0.2 has remained stable.

(C) 2019 The Author(s)

Published by S. Karger AG, Basel
\end{abstract}

\section{Introduction}

Idiopathic macular hole (MH) is a frequent disorder. It is usually isolated and requires a surgical approach. Wet age-related macular degeneration (AMD) is also characteristic for the central macular region, but those two disorders are rarely seen in coexistence. There is little 
information as to whether subretinal growth may cause MHs. It seems not to be very likely because otherwise most wet AMD patients with active choroidal neovascularization (CNV) would suffer from MH as well.

This paper describes a rare treatment mode of $\mathrm{MH}$ and active $\mathrm{CNV}$ in the same eye.

\section{Case Report}

A 72-year-old male patient was diagnosed with wet AMD in his left eye. Best corrected visual acuity (BCVA) in his left eye was 0.3. Optical coherence tomography (OCT) revealed CNV with subretinal fluid. Fluorescein angiography showed occult CNV in his left eye. The patient was put on anti-VEGF treatment with aflibercept (Eylea) every 4 weeks. Four days after the second injection he experienced a sudden drop in his visual acuity to the level of 0.05 . An additional OCT examination was done and revealed a full-thickness MH located in the center of the macula on the CNV. The anti-VEGF treatment was stopped and the patient was referred to a vitreoretinal surgeon for surgical consultation.

On examination by the vitreoretinal surgeon the right eye had 1.0 BCVA. Intraocular pressure was $14 \mathrm{~mm} \mathrm{Hg}$. Anterior segment changes were not clinically significant. The retina in the right eye was attached with no changes in periphery. OCT showed minor changes in right macula (small drusen only), and CNV was not present. No fluorescent angiography was performed at that point. BCVA in the patient's left eye was 0.05 . Intraocular pressure was $15 \mathrm{~mm}$ Hg. Anterior segment examination showed minor nuclear cataract. The retina was attached with no changes in periphery. On OCT examination CNV accompanied by MH was seen. The diameter of the MH measured on OCT was $420 \mu \mathrm{m}$ (Fig. 1).

It was obvious that the drop in BCVA in the left eye was due to the full-thickness MH formation. It was discussed with the patient that both wet AMD and MH require entirely different treatment approaches. It was decided to first operate on the $\mathrm{MH}$ and later continue the $\mathrm{CNV}$ treatment with anti-VEGF injections.

Due to the legal requirements in Poland (government reimbursement of surgical procedures), a cataract surgery followed by 23-gauge vitrectomy was performed. The vitrectomy was done in a typical way. The internal limiting membrane (ILM) was stained with Membrane Dual and peeled broadly up to the temporal vessel arcades; 20\% SF6 tamponade was used. Anti-VEGF treatment was not used at the time of the surgery. The patient was advised not to "raise his face to the ceiling" and no typical face down position was required. The MH was closed (Fig. 2). Visual acuity assessed 1 month after the surgery improved to 0.2 .

The patient was followed closely after the surgery and the first anti-VEGF injection was given 4 weeks after surgery. Further therapy with Eylea was scheduled every 8 weeks. Between January 2017 and March 2018 he was given a total of 9 Eylea injections and BCVA in his left eye remained at 0.2 .

\section{Discussion}

Idiopathic MH is a frequent disorder. The Beaver Dam Eye Study found full-thickness MHs to be prevalent in $0.3 \%$ of the population [1]. Another study by Kang et al. [2] showed that MHs affect women three times more often than men.

$\mathrm{MH}$ is a surgical disorder. However, different authors propose different surgical approaches. It is agreed that vitrectomy, ILM peeling, and gas tamponade should be performed 
to achieve MH closure. Recently a new technique - the inverted ILM flap technique - was described by Michalewska et al. [3].

In the case described in this paper, MH developed in an eye with CNV caused by wet AMD. There is no agreed theory how full-thickness MH develops. Gandorfer et al. [4] conducted histopathological examinations of ILMs removed during MH surgeries and proved that there was vitreous cortex insertion into the foveolar ILM in each specimen. They hypothesized that a partial posterior vitreous detachment with insertion of the vitreous cortex into the fovea might have created the macular tear which later was responsible for $\mathrm{MH}$ formation [4, 5]. Further macular change, especially increase in MH diameter, was - according to them - determined by the balance between retinal rigidity and tangential traction at the vitreoretinal interface $[4,5]$.

In the case described in this article, intravitreal anti-VEGF injection might have induced posterior vitreous changes. Geck et al. [6] proved that any drug injected into the vitreal cavity may induce posterior vitreous detachment. It is probable that in the case described in this article, retinal rigidity was already changed, which was caused by the CNV protruding from underneath. Additional intravitreal injection caused an imbalance between tangential tractions and retinal rigidity which resulted in $\mathrm{MH}$ formation. However, it is still difficult to determine why the changes occurred because otherwise all patients treated with injection would have had MHs.

Cases with coexisting $\mathrm{MH}$ and active $\mathrm{CNV}$ are not frequently described. There have been descriptions of the occurrence of MH and wet AMD in the same eye, but usually it was wet AMD that after years followed vitrectomy due to $\mathrm{MH}$, so these two disorders were not present simultaneously [7]. In 2004, Tabandeh et al. [7] published a retrospective trial where they described the frequency and morphology of subretinal neovascular membranes which developed in eyes after MH surgeries.

In the case described in this article, OCT examination showed typical idiopathic MH located on submacular neovascular membrane. There was direct communication between the subretinal space and the vitreal cavity, so it was impossible to assess the activity of CNV based on subretinal fluid level (Fig. 1).

In the case under discussion, vitrectomy was performed in a typical way. Semi-full vitrectomy was done, allowing for a big gas bubble to form. Filling $80-90 \%$ of the vitreous cavity with the $20 \%$ SF6 gas usually gives the adequate tamponade without the need of positioning the face down. Merely "no face up" is usually enough to achieve MH closure. The broadest possible ILM peeling was performed to mobilize the retina to close the hole. After successful vitrectomy, the $\mathrm{MH}$ was closed and the $\mathrm{CNV}$ remained under the retina.

Literature describing vitrectomy due to $\mathrm{MH}$ in eyes with coexisting CNV is rare. In 2001, Elsing et al. [8] described vitrectomy to close an MH which was performed with the concurrent removal of a subretinal CNV. The outcome of this surgery was poor. However, one needs to keep in mind that it was done before the anti-VEGF era.

In the described case, the surgery repaired the MH and wet AMD remained the disorder to be treated. The patient was on anti-VEGF treatment with Eylea at the time following the surgery. A typical dose of Eylea was used and no dose modification was done. No complication of the anti-VEGF treatment was noted. For 2 years following surgery, BCVA in the left eye reminded at 0.2 and no $\mathrm{MH}$ reopening was seen. 


\section{Conclusion}

An $\mathrm{MH}$ present in the same eye with active CNV is a rare finding. However, both disorders should be addressed in the otherwise typical way. Standard vitrectomy should be performed to achieve MH closure. Anti-VEGF treatment should be followed to stabilize the CNV.

\section{Statement of Ethics}

The treatment described in this paper complies with the guidelines for human studies; the research was conducted ethically in accordance with the World Medical Association Declaration of Helsinki. The patient gave his informed consent for the agreed treatment and for the publication of his medical data.

\section{Disclosure Statement}

The authors have no conflicts of interest to declare.

\section{Funding Sources}

The paper was fully founded by the author.

\section{References}

1 Klein R, Klein BE, Wang Q Moss SE. The epidemiology of epiretinal membranes. Trans Am Ophthalmol Soc. 1994;92:403-25.

2 Kang HK, Chang AA, Beaumont PE. The macular hole: report of an Australian surgical series and metaanalysis of the literature. Clin Exp Ophthalmol. 2000 Aug;28(4):298-308.

3 Michalewska Z, Michalewski J, Adelman RA, Nawrocki J. Inverted internal limiting membrane flap technique for large macular holes. Ophthalmology. 2010 0ct;117(10):2018-25.

4 Gandorfer A, Scheler R, Haritoglou C, Schumann R, Nentwich M, Kampik A. Pathology of the macular hole rim in flat-mounted internal limiting membrane specimens. Retina. 2009 Sep;29(8):1097-105.

5 Johnson MW, Van Newkirk MR, Meyer KA. Perifoveal vitreous detachment is the primary pathogenic event in idiopathic macular hole formation. Arch Ophthalmol. $2001 \mathrm{Feb} ; 119(2): 215-22$.

6 Geck U, Pustolla N, Baraki H, Atili A, Feltgen N, Hoerauf H. Posterior vitreous detachment following intravitreal drug injection. Graefes Arch Clin Exp Ophthalmol. 2013 Jul;251(7):1691-5.

7 Tabandeh H, Smiddy WE, Sullivan PM, Monshizadeh R, Rafiei N, Cheng L, et al. Characteristics and outcomes of choroidal neovascularization occurring after macular hole surgery. Retina. 2004 Oct;24(5):714-20.

8 Elsing SH, Postel EA, Gill MK, Jampol LM, Jaffe GJ. Management of eyes with both idiopathic macular hole and choroidal neovascularization. Retina. 2001;21(6):613-8. 


\section{Case Reports in Ophthalmology}

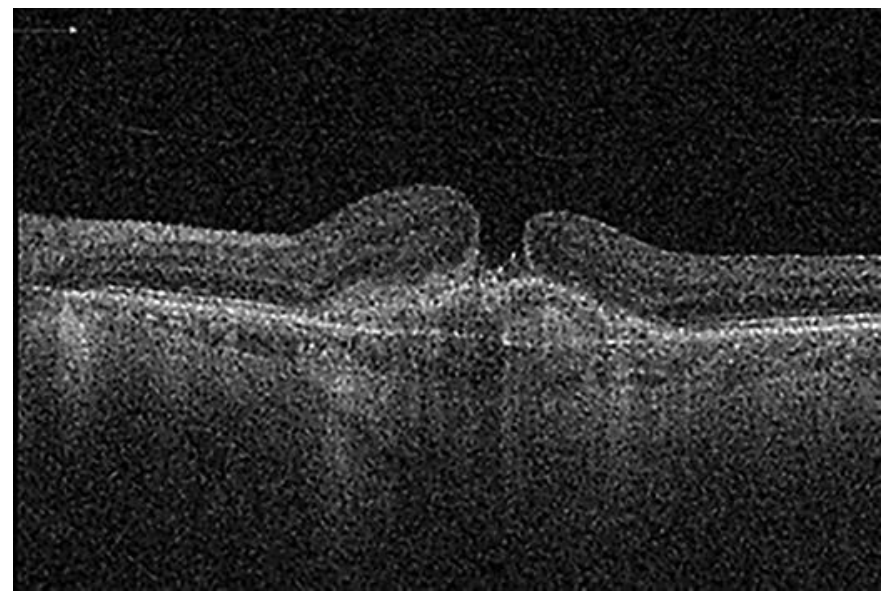

Fig. 1. OCT examination of the left eye. An MH and CNV are visible. Examination was performed on TOPCON Triton OCT.

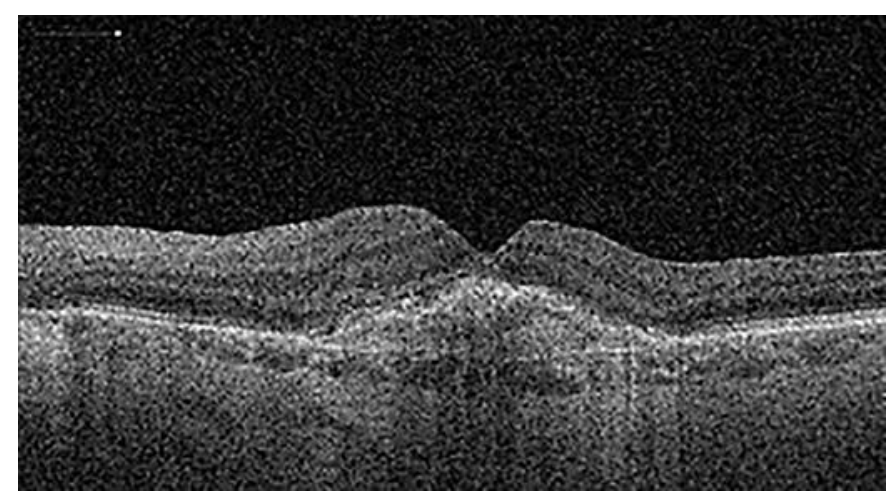

Fig. 2. OCT examination of the left eye after surgery. CNV is visible and the MH is closed. Examination was performed on TOPCON Triton OCT. 